\title{
The Use of Adaptive Traffic Signal Systems Based on Floating Car Data
}

\author{
Vittorio Astarita, Vincenzo Pasquale Giofrè, Giuseppe Guido, and Alessandro Vitale \\ Department of Civil Engineering, University of Calabria, Via P. Bucci Cubo 46B, 87036 Rende, Italy \\ Correspondence should be addressed to Vittorio Astarita; vittorio.astarita@unical.it
}

Received 13 February 2017; Accepted 11 April 2017; Published 11 May 2017

Academic Editor: Paolo Barsocchi

Copyright (C) 2017 Vittorio Astarita et al. This is an open access article distributed under the Creative Commons Attribution License, which permits unrestricted use, distribution, and reproduction in any medium, provided the original work is properly cited.

\begin{abstract}
This paper presents a simple concept which has not been, up to now, thoroughly explored in scientific research: the use of information coming from the network of Internet connected mobile devices (on vehicles) to regulate traffic light systems. Three large-scale changes are going to shape the future of transportation and could lead to the regulation of traffic signal system based on floating car data (FCD): (i) the implementation of Internet connected cars with global navigation satellite (GNSS) system receivers and the autonomous car revolution; (ii) the spreading of mobile cooperative Web 2.0 and the extension to connected vehicles; (iii) an increasing need for sustainability of transportation in terms of energy efficiency, traffic safety, and environmental issues. Up to now, the concept of floating car data (FCD) has only been extensively used to obtain traffic information and estimate traffic parameters. Traffic lights regulation based on FCD technology has not been fully researched since the implementation requires new ideas and algorithms. This paper intends to provide a seminal insight into the important issue of adaptive traffic light based on FCD by presenting ideas that can be useful to researchers and engineers in the long-term task of developing new algorithms and systems that may revolutionize the way traffic lights are regulated.
\end{abstract}

\section{Introduction, State of Art, and Contribution}

1.1. Introduction. In this paper, we introduce the use of FCD to regulate traffic lights. It must be noted that the approach of this paper goes in a different direction from many recent research papers in the adaptive traffic signal sector. Consensus is established on the fact that new connectivity technologies with Vehicle-to-Infrastructure, infrastructure to vehicle, and vehicle-to-vehicle communications will pave the way for new adaptive traffic signal systems. There is no agreement on how this will happen and there are many different proposals tested in simulation.

Originally, traffic adaptive systems were based on the traditional idea of sensors that can count vehicles approaching intersections. Recent developments of this kind of idea have introduced wireless sensors that can be placed easily, where necessary, further extending this concept. A recent example is in Tubaishat et al. [1] where adaptive traffic light control is obtained with a network of wireless sensors deployed on the entering/exiting lanes of the intersections.
Instead, the idea of this paper is to use information coming from the vehicles that would localize themselves through GNSS (or other terrestrial localization radio systems) and then communicate positions and speeds to a central control unit.

Moreover, another recent concept has been discussed in a new stream of literature where recent works have been centered on using Advisory Speed Limit (ASL) control strategies. An example is Stevanovic et al. [2] or in Ubiergo and Jin [3] where Vehicle-to-Infrastructure communication is the base underlying the application of an ASL strategy with the effect of smoothing vehicle trajectories and reducing total travel time.

ASL technology is very promising given the new connectivity technologies that will be introduced in newly produced cars.

It is the opinion of the authors that even though ASL systems are useful there is more collective advantage to proceed mainly by regulating traffic lights according to traffic 
demand than changing traffic driving patterns and demand according to preestablished traffic signal plans.

The best solutions that will take full advantage of new connectivity technologies will be mixed technologies where ASL will be superimposed on adaptive traffic signals from FCD as envisioned in Le et al. [4].

1.2. State of the Art and Contribution. Now, in all the applications deployed on the field, all of the real time information obtained from "instrumented" vehicles as traffic probes comes from the coupling of the mobile phone data network and the GNSS system. This real time information has been used to give back user traffic information and give road guidance in systems such as Tom Tom, Google Maps, Here Maps, iGO, Ovi Maps, and Waze. Insurance companies have used the same system with dedicated devices applied to vehicles to obtain information useful to assess driving risks associated with distance traveled and other dynamic parameters. In all the above systems, information obtained from the GNSS system is transferred via wireless mobile phone network directly to a central database.

FCD have not been widely used yet to implement traffic control operations since information in most cases is not in the hands of city and other road administrations and since where such information was obtained by city administrations there has not yet been a sufficient and consistent diffusion of mobile "city" phones applications among the public. For example, cities like Santander have started to experiment in the framework of "Smart City" projects with a dedicated mobile phone application; the experiment has shown that it is still necessary to reach a large-scale diffusion of these dedicated mobile phone applications to make them effective.

Whether or not those experiences show real flaws in traffic signal light systems they hint that it is becoming urgent to better regulate traffic light systems. Moreover, traffic control can take advantage of the information obtained from floating car data (FCD) that can become an essential source for traffic regulation.

As stated in Astarita et al. [5], the whole concept of floating car data (FCD) is based on the collection of localization data, speed, direction of travel, and other information from vehicles. FCD can be obtained by having vehicles instrumented with a Global Navigation Satellite System (GNSS) receiver and a radio data connection. The mobile phones that drivers and passengers carry in vehicles, in most cases, already potentially have both systems and have the advantage of being already deployed on practically every vehicle.

For this reason, traffic regulation could use the information coming from road user mobile phones once specific software is used. Smartphones can become perfect traffic probes once drivers are given the right "killer application" that would convince them to accept this system as beneficial. While, in the future, automotive industries will provide "fully connected" vehicles that also will be able to act as FCD, at the moment (2017) all FCD real world implementation come only from "instrumented" vehicles based on just mobile phones.

Among the many researches on obtaining traffic data from instrumented vehicles as traffic probes, some have been focalized on the use of specific Radio-frequency identification (RFID) transponders like Ban et al. [6] and Wright and Dahlgren [7]. The drawback to these systems is that they need infrastructure expenses to set up readers. Some have been experimenting specifically using mobile phone data to evaluate traffic conditions. Among them early works have been considering the possibility of using just mobile phone data without GNSS system data: Astarita et al. [8], Ygnace et al. [9], Bolla and Davoli [10], Bolla et al. [11], Astarita and Florian [12], Rose [13], Astarita et al. [14], Sohn and Hwang [15], and Calabrese et al. [16].

Other works have been focused on obtaining road pavement conditions and fuel consumption estimation from mobile phones: Astarita et al. [17], Mohan et al. [18], and Astarita et al. [19]. Dailey and Cathey [20] proposed the use of data coming from transit vehicles to estimate traffic speeds. Axer and Friedrich [21] investigated the use of low frequency FCD to estimate the level of service on traffic networks. FCD data have also been used to establish unsafe driving behaviors in Axer and Friedrich [22]. Other works on traffic safety based on FCD are Biral et al. [23], Vaiana et al. [24], and Guido et al. [25]. Kerner et al. [26] showed that $1.5 \%$ of FCD penetration rate can be enough to detect an incident in a traffic center with a probability of $65 \%$.

In other research works, the possibility of using smartphones with satellite localization and wireless Internet data transmission has been considered. First works of this kind investigated the penetration rate necessary to achieve good results for traffic information systems. Ygnace et al. [9] established that $5 \%$ of the car instrumented with GPS sensors would be enough to estimate link travel times on a freeway network with an accuracy of 95\%. Ferman et al. [27] showed that a real time traffic information system based on probe vehicles is feasible and should work for freeways at penetrations over $3 \%$, while other roads would require more than $5 \%$. Other works have centered on real implementation in the field of combined GNSS and data transmission, such as Herrera et al. [28] and Guido et al. [29, 30].

A whole sector of research generated from the idea of sampling traffic by detecting mobile phone radio signals among traffic vehicles. The idea of using radio receivers was envisioned in the patent Astarita et al. [8] and has produced real implementation in the field in 2008 using Bluetooth protocol to detect the Bluetooth mobile phone radio signals with an external active Bluetooth device. Results of early works in this field were presented starting in June 2008 by Wasson et al. [31] and Young [32]. Other interesting papers on the subject are Barceló et al. [33], Barceló et al. [34], and Bachmann et al. [35].

Mobile phones GNSS data were used to evaluate adaptive traffic signal systems in Hu et al. [36]. Mobile phones GNSS were used also to obtain information on signal timings by Axer et al. [37] and Barthauer and Friedrich [38], in which the information on signal data is used for driving assistance functions such as green light optimal speed advisory and countdown signal showing the remaining time before the next switch in the signal.

The use of FCD data to regulate traffic signals is studied in Saust et al. [39] where a cooperative system is presented 
in which both the infrastructure and the driver behavior is adjusted so that a double optimization is reached. Also in the project Colombo [40, 41], traffic signal regulation is envisioned from Vehicle-to-Infrastructure communication. Gradinescu et al. [42] propose using vehicle-to-vehicle communication and using microscopic simulation to evaluate the proposed sophisticated adaptive control system.

This paper investigates the basic concepts of FCD Adaptive Traffic Lights (FCDATL) because of the following three mega trends:

(1) The implementation of Internet connected cars with global navigation satellite (GNSS) system receivers and the autonomous car revolution: vehicle-to-vehicle (V2V) and Vehicle-to-Infrastructure (V2I) technologies will become reality very soon as both carmakers and public authorities in both Europe and United States are pushing to provide a framework that can help safer and more sustainable driving. There is an ongoing international deployment of new technologies between the European Commission and US Department of Transport (USDOT) intended to create collaboration on cooperative vehicle safety, mobility, sustainability application, research projects, and international intelligent transportation system (ITS) standards [43]. Moreover, companies such as Tesla, other carmakers, and Google are investing in autonomous vehicles that can be driven with reduced, or completely without, driver participation.

(2) The mobile cooperative Web 2.0 and the extension to connected vehicles: the mobile web 2.0 is a current innovation consisting of a series of disruptive services, which will form new markets and shift consolidated equilibriums. These kinds of application are growing at a rapid rate effectively tying the web 2.0 with smart mobile devices and creating a type of services that use mobility and satellite data and at the same time are easy and immediate and of ubiquitous use. The main forces that drive the deployment of such applications are the spread of mobile Internet; the use of satellite data; social networks; the creation of user-generated content (User generated web 2.0).

These services point to the future based on some common aspects such as

(i) openness: open standards, open-source development and open access;

(ii) the use of specially developed smart mobile devices applications rather than the use of web pages;

(iii) affordable mobile phone network access for everyone of contents and services (such prices are a very recent expression of the market);

(iv) choice for the user to communicate and share experiences with others (social interaction);

(v) user-generated mobile content. This information can have a better acceptability as provided by other people who share the same positive experiences;

(vi) services based on the coupling of satellite location and mobile web 2.0 applications and smart devices, in other words "aware" apps that know where you are and are able to provide you with what you want in this place and at this time, using the satellite sensors integrated seamlessly into applications;

(vii) mobile web 2.0 and digital convergence (the mobile web 2.0 becomes a driver for the digital convergence).

Mobile cooperative web 2.0 can make possible a large deployment of cooperative-ITS Systems where a great amount of information can become useful for traffic control operations and transportation system management. Road operators, infrastructure, vehicles, their drivers, and other road users will be able to cooperate to deliver the most efficient, safe, secure, and comfortable journey.

(3) Sustainability of transportation in terms of energy efficiency, traffic safety, and environmental issues.

In the following, a classification of the different possible light regulation systems based on FCD is presented (after having introduced the concept of FCD Adaptive Traffic Lights (FCDATL))

FCD Adaptive Traffic Lights (FCDATL) systems are conceptually different from that of any other adaptive traffic light control system as they are based on the acquisition of the sequence of positions and velocities obtained from satellite receivers of instrumented vehicles. Traditional adaptive traffic signals are based on measures of traffic flows and speeds at localized measuring stations. Using FCD means that the vehicle itself will communicate position and speed with continuity to the control center.

With Adaptive Traffic Lights (FCDATL) the ideal situation is that in which all vehicles are "instrumented"; the regulation system would have available all the positions of all the vehicles and the relative speed on the network allowing for an optimal traffic signal regulation which would never be possible with the traditional systems. In this case, the quantity of information would be better than what could be obtained with traditional counting systems, guaranteeing better control results, although new algorithms would be needed to regulate traffic lights according to all vehicle trajectories measured in real time.

In real intermediate situations, only a part of all vehicles would be instrumented and traffic light regulation would have to operate on the basis of only some of the vehicle trajectories.

The algorithms needed to regulate traffic lights according to such a mixed data set would serve two different systems:

(1) Algorithms for "Mixed FCD Adaptive Traffic Lights" (MFCDATL) where trajectories of instrumented vehicles are used in conjunction with information coming from traditional measurement systems (such as loop detectors);

(2) Algorithms for "Pure FCD Adaptive Traffic Lights" (PFCDATL) where only trajectories of instrumented vehicles are used in establishing traffic light optimal phases.

In this second case, the algorithms that serve PFCDATL can use vehicles positions and speeds and estimate traffic flows for each turning maneuver on the basis of "instrumented vehicles" counts or be totally pure, in the sense that they are based only on vehicle positions (queue lengths). 
Traffic flow values that are needed for traffic light regulation can be estimated on the basis of the methodology described in Astarita et al. [14] or other methodologies just from "instrumented" vehicles counts. Traffic turning measurements thus obtained are an estimate of total traffic turning maneuvers where accuracy is determined, of course, by the ratio between the number of "instrumented" vehicles and the total number of vehicles.

Mixed FCD Adaptive Traffic Lights systems (MFCDATL) would have the advantage of using more information than the traditional system, guaranteeing a better control. They would also have the same disadvantages as traditional systems such as the cost of fixed infrastructures for traffic measures, whereas PFCDATL would have the advantage of not using any costly fixed infrastructures instead relying only on the GNSS localization system and the mobile wireless Internet already deployed in every developed country.

Before developing a system, investing money and deploying it on the road it would be useful to develop dedicated algorithms and test them with microsimulation to assess results with different percentages of "instrumented" vehicles.

This paper follows this path, developing a traffic simulation analysis framework on the basis of which it would also be possible to assess a business plan and establish the convenience of investing in a specific system of this kind.

In detail, this paper presents the advantages of using FCD data for regulating traffic signals taking into consideration different FCD penetration rates, different traffic flow patterns, and effects on different end-users. Microsimulation on a simple test network has been used to make an assessment on a simple greedy algorithm used to optimize traffic phases. The advantages of using these kinds of system are assessed from the point of view of subscribers and overall network travel time reduction according to various subscriber rates in the driver population.

A simple greedy algorithm for adaptive traffic signal optimization based on FCD is in fact presented and analyzed. The algorithm has been tested on microsimulated scenarios to assess the performance with varying FCD penetration rates for both "instrumented" vehicles and "noninstrumented" vehicles.

An "instrumented" vehicle is considered a vehicle on which there are both a GNSS localization system and a radio data transmission system.

The microsimulation model used in this work is able to generate vehicles randomly according to a given rate of FCD. Moreover, it is able to simulate both the movement of vehicles and the error in the GNSS localization of instrumented vehicles to reproduce more realistically what would happen in reality. The localizations of "instrumented" vehicles are also elaborated with a map-matching algorithm to fix some position errors and to obtain the information necessary to regulate the traffic light.

The adopted procedure could be used as a framework useful to researchers and engineers in the long-term task of developing new algorithms and systems that will revolutionize the way traffic lights are regulated.

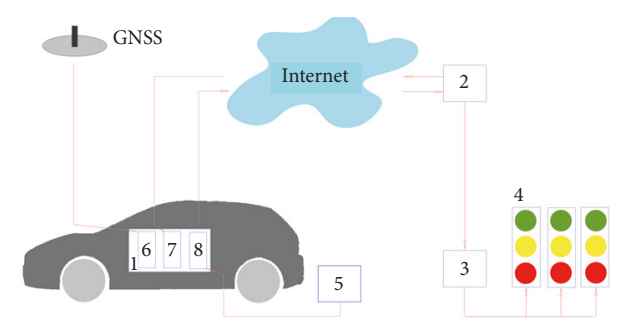

FIGURE 1: General structure of the proposed system.

\section{Proposed Method}

2.1. A New Cooperative Intelligent Transportation System for Traffic Light Regulation. This section presents a possible new cooperative intelligent transportation system for traffic light regulation. It will be tested as explained below using a general methodology applicable also to similar yet different systems. In fact, it must be noted that the system here presented is just one of the many different systems that can be designed and implemented. The considerations presented in the following sections of this paper apply not only to the system presented in this section but also to all other possible systems that share the same concept of using FCD data to regulate traffic lights.

The presented system has the advantage that it could be implemented right away on the road with a simple mobile phone application or also in the future with connected vehicles in the framework of Vehicle-to-Infrastructure communication.

Interested drivers would subscribe to the service and install a phone application on mobile devices with GNSS capabilities (such as GPS and Galileo) or buy a "connected car" with this feature embedded. The mobile phone application or the "connected car" would send the position and speed of the vehicles continuously to a central server via the standard mobile phone radio data network (or any other dedicated radio data network). This would allow the traffic light regulation system to be managed knowing the position of service subscribers and traffic lights to be regulated according to this.

It is not the scope of the paper to discuss the moral or political convenience of making such a system a payment system or to suggest different payment options and/or green priorities for different users. Although it should be noted that technically the system would also allow prioritizing of vehicles according to payment schemes. Moreover, a system of this kind would allow vehicles such as ambulances or priority vehicles to move faster on the network comparing to other vehicles.

The proposed general system (a detailed description of a complete implementable system of this kind can be found in Astarita et al., [5]) is composed (as depicted in Figure 1) of the following modules:

(1) "Instrumented" vehicles (1) equipped with satellite receiver position system (GNSS) (6) and/or local radio receiving system (8), used together or as an alternative to the satellite system for vehicles location; wireless data communication device (Internet) based 
on wireless mobile data network or wireless local area data network (7);

(2) Central server (2) for data collecting and processing and for establishing the signal phases that are communicated to the connected controller unit (3) that will activate traffic lights (4);

(3) Traffic light controller;

(4) Traffic lights;

(5) Local radio signals emitters (5) that can be appropriately placed on the side of road network allowing the localization of instrumented vehicles when satellite localization is not usable.

In the proposed system, the central server (2) receives data on positions and speeds of instrumented vehicles (1). The central server then processes the data to determine the best traffic light plan to be transmitted to the control units (3).

The system can combine information obtained from FCD with other vehicular traffic information obtained with other traditional traffic counts systems (magnetic loop detector, microwave radar sensors, etc.).

Moreover, the adaptive traffic light management system here presented is able also to guarantee the green phase to certain categories of vehicles also according to a logic of payment subscription and provide driving information via mobile devices and via on board instruments, taking into account the timing of planned traffic lights phases at intersections.

\subsection{The Use of Local Radio Signals for Car Positioning. Local} radio signals can be used to localize vehicles where the satellite signal is too low due to the presence of tall buildings (urban canyons). The idea is exactly the contrary of what is currently done today with traffic sensors. Traffic sensors are deployed to detect vehicles on the road. With local radio signals, it is the vehicle itself that has a radio receiver able to read a local radio signal. Once the signal is read the vehicle position is determined on the base of the known position of the radio emitter.

Local radio transmission systems like Wi-Fi can be used although they require an external power source and higher setup and maintenance costs. For this reasons the use of Bluetooth Low Energy (BLE) signals from battery-driven beacons seems more promising. BLE is a recent technology that is emerging as a standard for indoor positioning. BLE beacons (or iBeacons) are extremely cheap and with a long battery life and they make possible an inexpensive deployment near road intersections.

In this paper, the idea of using BLE beacons to localize vehicles on the road (instead of the common indoor use [44, 45]) is introduced [46] and discussed for the first time.

Since there is no literature discussion on the pros and cons of using iBeacons (or other local radio emitter) coupled with smartphones or instrumented vehicles, instead, there is extensive literature $[47,48]$ on how to use iBeacons to calculate approximate indoor distances and estimate indoor location; we present briefly the results of a first preliminary experimentation with iBeacons and vehicles.
The experiments that we carried out have shown that standard mobile phones with Bluetooth function and carried inside vehicles are able to catch the signal of iBeacons positioned at the side of the road up to a speed of $40 \mathrm{~km} / \mathrm{h}$.

While the preliminary results above presented may not be definitive on whether it would be possible to carry out normal traffic flow detection with iBeacons, they are very promising for the possibility of using the iBeacon to establish whether vehicles are waiting in queues at a signalized traffic intersection as described in the above presented system.

It must be noted that in the envisioned system the smartphones or the connected vehicles must have a dedicated software able to identify the vehicle position and communicate to the central control server.

\section{Testing the Proposed Method}

3.1. A Framework for the Assessment of the Proposed Systems. The proposed system was tested using microsimulation on a single intersection, making comparisons between performances of the proposed system (using mobile phones as sensors) and a traditional fixed time signal cycle.

The TRITONE microscopic traffic simulator [49] was used with a dedicated module for postprocessing of vehicles trajectories and positions. This module was developed to introduce the GNSS error in the estimation of positions that would be met in a real environment. The positions with random errors were then analyzed with a map-matching algorithm obtaining estimates that were fed into the adaptive traffic signal algorithm.

Numerical results were obtained after 50 repetitions of every different scenario. Scenarios have been estimated from the point of view of both subscriber advantages and overall network travel time reduction as a function of different subscriber rates in the driver population.

The operating logic and the instruments used by the traffic light control are different from those of any other existing traffic light control system because the system is based on the acquisition and processing of the sequence of positions and speeds obtained from the satellite positioning receivers of instrumented vehicles. The system, in fact, can not only allow priority to be given to some categories of users in the allocation of green times, it can also send driving instructions according to the foreseen green times provided by the system.

In the following sections, the four modules that compose the simulation test bed are described in detail (Figure 2):

(1) The microsimulation of an isolated intersection with a random generation of instrumented vehicles according to an established penetration rate $\varphi$;

(2) GNSS error generation module: in this module, positions of the moving instrumented vehicles generated by the microsimulation module are made realistic by assigning a random error with given variance along the $x$ - and $y$-axis;

(3) Map-matching algorithm: the coordinates of instrumented vehicles that have been corrected inserting an 


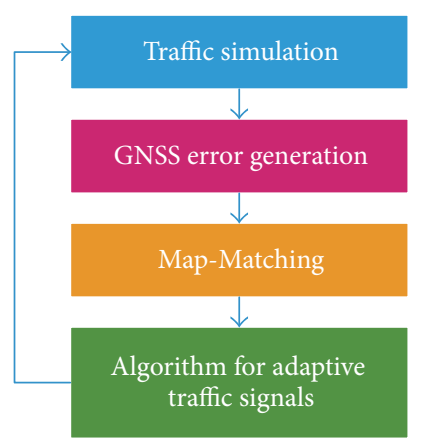

FIGURE 2: General simulation framework to assess performances of the adaptive signal control algorithms.

error are elaborated to match the road system so as to assign each vehicle to a possible position over the road network;

(4) The algorithms for traffic light control. In this paper, two algorithms are presented and analyzed that can be applied to two different intersections layouts.

3.2. The Microscopic Simulation Model. To carry out the above described simulation framework, the GNSS error generation module, the map-matching algorithm, and the algorithm for traffic light control were implemented on the microscopic traffic simulation model TRITONE. The choice of TRITONE was based on its versatility. In fact, TRITONE is able to simulate both freeways and urban road networks also, TRITONE makes possible to modify simulation procedures and also evaluate traffic safety performances. TRITONE was produced with the specific intention of reproducing Intelligent Transportation Systems such as ATMS and ATIS, performing modeling of both traffic and traffic safety performances. TRITONE is a classic microsimulation model where the traffic performances are obtained reproducing individual vehicle movements. Moreover, in TRITONE it is also possible to introduce macroscopic traffic flow relationships by using specific car following models based on macroscopic link characteristics such as free flow speed and capacity. The microsimulation model INTEGRATION [50] that in the past was considered mesoscopic by some researchers has inspired the combined use of individual vehicles and macroscopic flow theory.

Moreover, the use of TRITONE allowed automatic simulation of a sequence of many different traffic scenarios, each reproduced with 50 repetitions.

More information on TRITONE can be found in Astarita et al. [51].

3.3. GNSS Error Generation Module. The simulation of the satellite location is done by inducing an error in the position of the vehicle, as the real sensors are not able to evaluate the position with certainty but establish a confidence range. To assign the error to the GNSS position of the vehicle given by the simulator, it is considered that any given point on the
GNSS sensor is in reality at any point in the domain whose radius is defined by the accuracy parameter (Figure 3 ).

Studies carried out by Steffen et al. [52] established that the position provided by the GNSS sensor via a reference to polar coordinates where the radius is a random value that is associated with a normal distribution with mean 0 and standard deviation 25 meters can be simulated:

Radius is normal distribution (mean $=0$, dev.std $=$ 25).

Angle is random value between 0 and 360 .

The coordinates of the vehicle such as obtained from the GNSS receiver will be

$$
\begin{gathered}
X_{\mathrm{GNSS}}=X+\text { radius } \cdot \cos (\text { angle }) \\
Y_{\mathrm{GNSS}}=Y+\text { radius } \cdot \sin (\text { angle }) .
\end{gathered}
$$

3.4. Map-Matching Algorithm. This procedure consists in bringing the location point obtained from the GNSS sensor to the most likely position compatible with real road arcs. The operation consists of two parts, the closest arc choice and the location point over the chosen arc. The arc choice is obtained by evaluating the consistency of the vehicle trajectory with arc characteristics (Figure 4). Regarding the vehicle trajectory, only the last five detected coordinates are taken into consideration.

To establish the right arc for a vehicle, four parameters are examined:

(1) The arc angle (always positive, which varies from 0 to $2 \pi)$ : this parameter is used to assess the congruence between the direction of the vehicle and the road direction, because the network arcs are always considered unidirectional;

(2) The distance between the vehicle and the arc center: this parameter is useful to assess which is the closest arc;

(3) The perpendicular distance GM between the G point obtained by GNSS positioning and the various arcs around point G: this is calculated using the equation that describes a perpendicular line between the point and each arc;

(4) The average speed of the road. To assess the correct road in case there are different roads with different priorities (urban streets, highways, etc.).

Every GNSS vehicle position is assigned to a single arc according to all these four parameters.

Once one GNSS position is assigned to an arc the right position on the arc is be established by simple geometric projection.

3.5. An Algorithm for Adaptive Traffic Signals. A simple greedy algorithm has been developed and applied in the following case study for the optimization of traffic light phases given FCD data. The algorithm evaluates the number of instrumented vehicles that are present on every approaching 


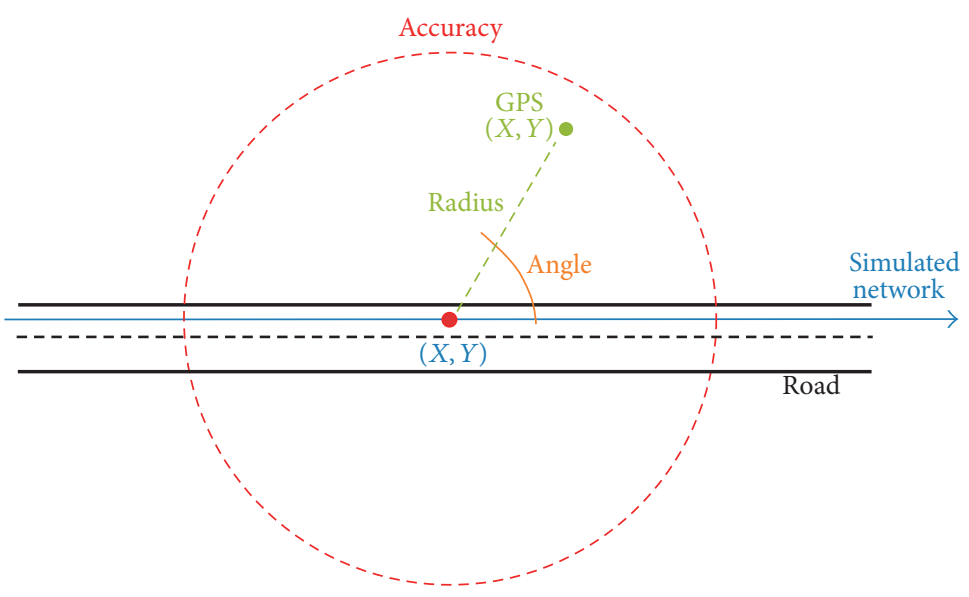

Figure 3: Simulation of GPS coordinates.

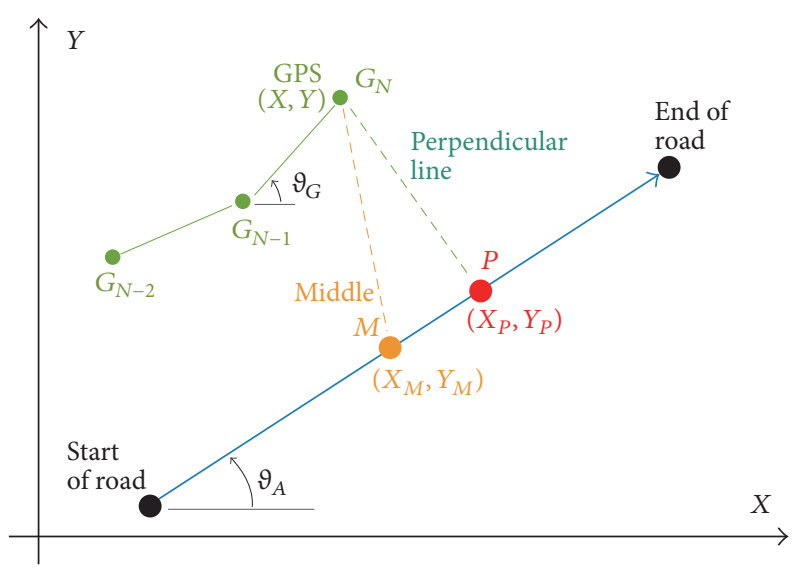

FIGURE 4: Establishing the most likely arc.

street and assigns green to the approaches with priority to the approach that has more instrumented vehicles. The green time assigned to every approach depends on the evaluated length of the queue, which is established on the base of the position of the last instrumented vehicle in queue.

Having evaluated the position in the queue of the last vehicle the green time allocated to the relative phase is just what is necessary to let the last instrumented vehicle exit the intersection.

If there are no instrumented vehicles at the intersection the algorithm falls back into a preestablished fixed time cycle. In any case, the algorithm assigns a minimum green of 4 seconds for every phase (according to traffic light UK rules). It must be noted that the proposed optimization single intersection algorithm can be extended to a whole network in the case in which every intersection has one single lane for approach.

This algorithm is just a first attempt to evaluate the system concept of regulating traffic lights with FCD data. In the following part, some limitations of this first attempt algorithm will be discussed.

The control algorithm is presented in the flow chart depicted in Figure 5.

\section{Case Study}

4.1. Intersection with Single Lane for Each Approach and a Simple Greedy Optimization Algorithm for Adaptive Traffic Signals from FCD. A simple single intersection with one single lane for each approach has been simulated as the first scenario to test the system procedure (Figure 6).

To assess the above described algorithm performances three main cases were investigated and in any studied case traffic volumes have been simulated according to 5 different directional distribution flow patterns. This gives a total of 15 scenarios that have been simulated 51 times (varying the distribution of instrumented vehicles with a $2 \%$ step). Each traffic scenario has been simulated 50 times to reduce effects due to the random variability of the microsimulation approach.

In other words, 765 scenarios have been simulated. Each scenario was simulated 50 times for a total of 38.250 traffic simulations that have been carried out with the microsimulation software TRITONE on the intersection in Figure 7.

The three main cases are

(1) Case A, with a total demand of $480 \mathrm{v} / \mathrm{h}$ according to traffic patterns A and optimum traffic light cycles for the given traffic flows;

(2) Case B, with a total demand of $240 \mathrm{v} / \mathrm{h}$ and optimum fixed traffic light cycle for the given traffic flows;

(3) Case C, with a total demand of $240 \mathrm{v} / \mathrm{h}$ and not optimal fixed traffic light cycle.

Case $\mathrm{C}$ corresponds to those situations in which the traffic light cycle was calibrated for managing traffic flows that have been measured once on the field and that are different from the real traffic that actually circulates (often happens unfortunately in reality in Italian intersections).

In Cases $\mathrm{A}, \mathrm{B}$, and $\mathrm{C}$, as above noted, traffic volumes have been simulated according to 5 different flow patterns in order to consider different direction repartition: 50/50, 40/60, 30/70, 20/80, and 10/90 (Table 1).

For each of the five directional flow distributions a fixed static traffic light cycle was calculated according to the 
TABLE 1: Traffic flows for each simulated scenario and O/D couple in veh/h.

\begin{tabular}{|c|c|c|c|c|c|c|c|c|c|c|c|}
\hline \multirow{2}{*}{$\begin{array}{l}\text { Orig. } \\
\text { node }\end{array}$} & \multirow{2}{*}{$\begin{array}{l}\text { Dest. } \\
\text { node }\end{array}$} & \multicolumn{5}{|c|}{ Traffic pattern with total demand $480[\mathrm{VPH}]$} & \multicolumn{5}{|c|}{ Traffic pattern with total demand $240[\mathrm{VPH}]$} \\
\hline & & $50 / 50$ & $60 / 40$ & $70 / 30$ & $80 / 20$ & $90 / 10$ & $50 / 50$ & $60 / 40$ & $70 / 30$ & $80 / 20$ & $90 / 10$ \\
\hline 2 & 3 & 40 & 48 & 56 & 64 & 72 & 20 & 24 & 28 & 32 & 36 \\
\hline 2 & 4 & 40 & 48 & 56 & 64 & 72 & 20 & 24 & 28 & 32 & 36 \\
\hline 2 & 5 & 40 & 48 & 56 & 64 & 72 & 20 & 24 & 28 & 32 & 36 \\
\hline 3 & 2 & 40 & 48 & 56 & 64 & 72 & 20 & 24 & 28 & 32 & 36 \\
\hline 3 & 4 & 40 & 48 & 56 & 64 & 72 & 20 & 24 & 28 & 32 & 36 \\
\hline 3 & 5 & 40 & 48 & 56 & 64 & 72 & 20 & 24 & 28 & 32 & 36 \\
\hline 4 & 2 & 40 & 32 & 24 & 16 & 8 & 20 & 16 & 12 & 8 & 4 \\
\hline 4 & 3 & 40 & 32 & 24 & 16 & 8 & 20 & 16 & 12 & 8 & 4 \\
\hline 4 & 5 & 40 & 32 & 24 & 16 & 8 & 20 & 16 & 12 & 8 & 4 \\
\hline 5 & 2 & 40 & 32 & 24 & 16 & 8 & 20 & 16 & 12 & 8 & 4 \\
\hline 5 & 3 & 40 & 32 & 24 & 16 & 8 & 20 & 16 & 12 & 8 & 4 \\
\hline 5 & 4 & 40 & 32 & 24 & 16 & 8 & 20 & 16 & 12 & 8 & 4 \\
\hline
\end{tabular}

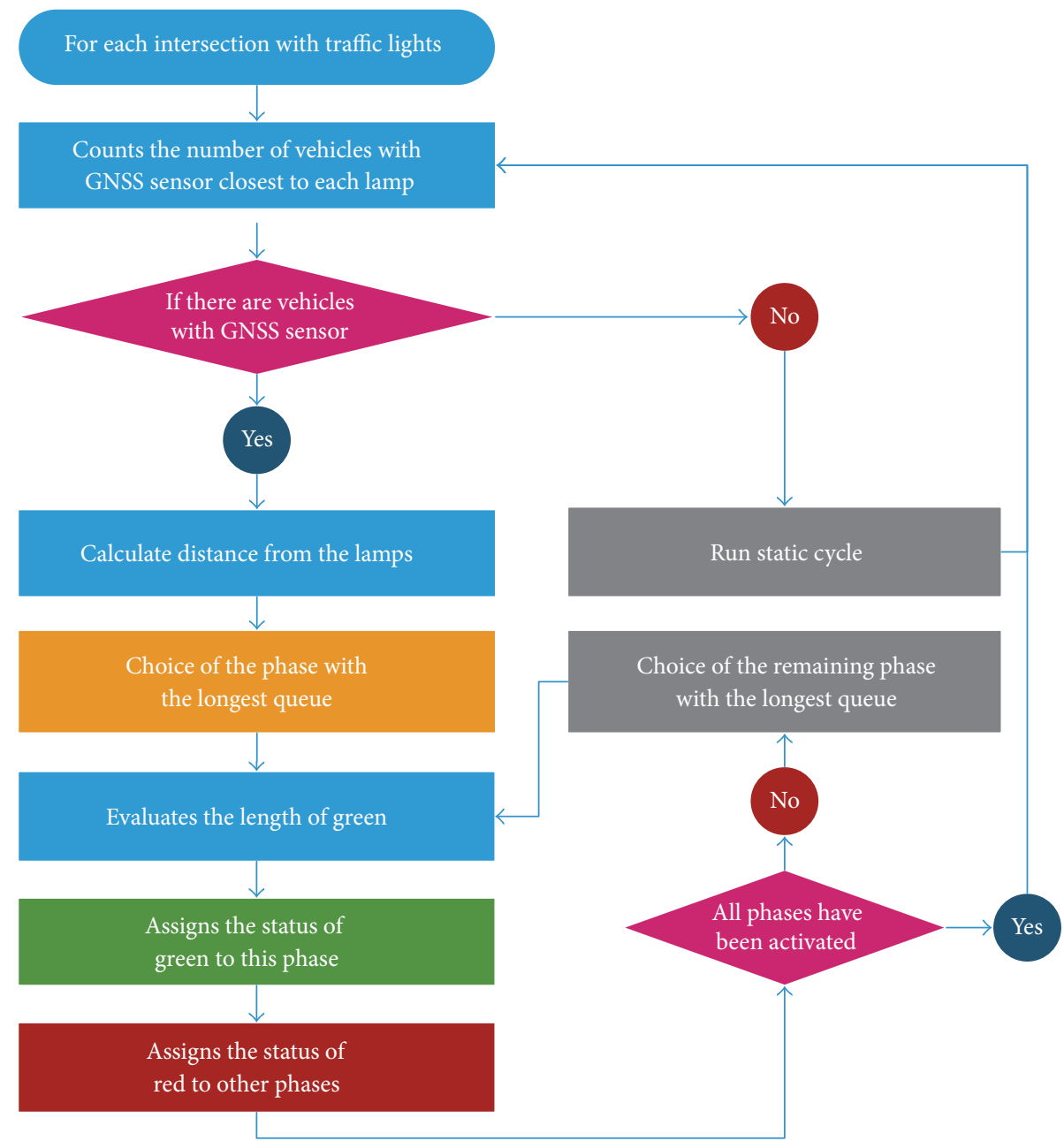

FIGURE 5: Greedy algorithm for single lane intersections.

indications of the highway Capacity Manual. An example is shown in Figure 7.

Scenarios have been estimated primarily to establish differences from the point of view of the two different type of users (instrumented and not instrumented vehicles) and to check the overall network travel time reduction as a function of different subscriber rates in the driver population.

Overall performance in Cases A, B, and C are presented in Figures 8, 9, and 10 that show total travel time of all vehicles both instrumented and not instrumented with varying 


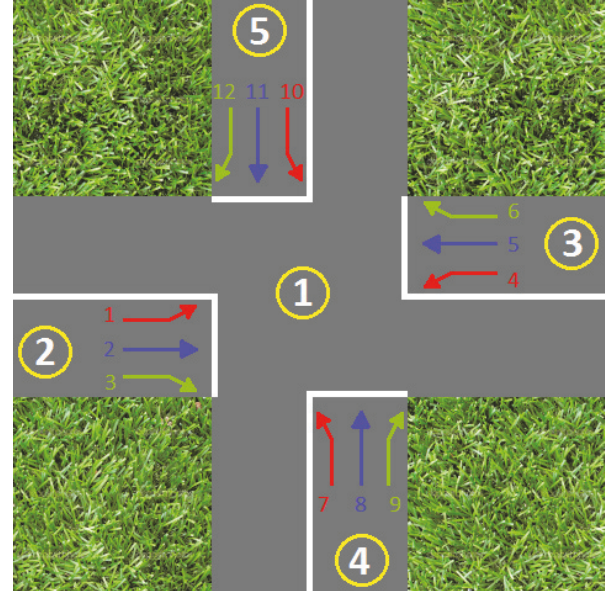

FIGURE 6: The simulated intersection with single lane for each approach.

percentage of "instrumented vehicles." The four green phases share the total available time according to HCM optimized prefixed time plan when the optimization algorithm is not applied in the case that there are no instrumented vehicles at the intersection or when the percentage of "instrumented" vehicles is equal to zero.

Figure 8 shows the total travel time for the intersection of case A. A fixed signal plan has been fixed according to HCM procedure. Traffic 50/50 means that northbound/southbound traffic is equal to eastbound/westbound traffic.

In Figure 9 is depicted the total travel time for the intersection of case B with total traffic flow at around 50\% of intersection capacity

In Figure 10 is depicted the total travel time for the intersection of case C with total traffic flow at around 100\% of intersection capacity and a fixed signal plan which has a longer cycle (wrong cycle) established as if the intersection was near to saturation.

Results show that the system is able to perform the same or better than a fixed time plan with an overall convenience of the system that increases with a higher instrumented vehicles percentage. Obviously in case $\mathrm{C}$ where traffic lights are not regulated at the optimal fixed time plan the advantage of the system is most relevant. Already at $30 \%$ of instrumented vehicles rate there is an overall reduction in travel times.

The travel time performance of not instrumented vehicles is presented in Figures 11, 12, and 13.

The vehicles, which are not instrumented, are only very slightly slowed down.

The relative advantage (or disadvantage for not instrumented vehicles) for instrumented vehicles relative to not instrumented vehicles is presented in Figures 14, 15, and 16.

\section{Conclusions}

This paper provides insights into the important issue of traffic light regulation based on FCD data coming from smartphones. The paper intends to provide ideas that can be useful to researchers and engineers in the task of developing new algorithms that making use of FCD can revolutionize the way traffic lights are regulated.

The methodology, which was presented in this paper and used to assess results of FCDATL, has showed some important key concepts:

(i) With a low number of subscribers (as, e.g., in the launch phase of such systems) the first drivers using the system would be given green priority on other drivers;

(ii) When the percentage of subscribers reaches around $35 \%$ of all drivers the system would operate more democratically and very efficiently in traffic regulation because it would guarantee optimal cycle lengths and phase times for all users. These results would be obtained by using the subscriber base to estimate the position of all vehicles in the network. Users of the system would be used as a sample for the whole population of cars.

The above described preliminary results of simulations on a single intersection are very interesting since they show a pattern in travel time reductions that justifies the implementation of a new cooperative intelligent transportation system for traffic regulation that would benefit "instrumented" vehicles, noninstrumented vehicles, and the city administrations fulfilling these two objectives:

(1) Regulating traffic signals and also informing drivers on road conditions ahead by using Floating Car Data (FCD);

(2) Convincing drivers to accept this system as beneficial voluntarily so becoming part of a successful cooperative system.

It is opinion of the authors that the implementation of such a system, in fact, could be the "killer smartphone application" that would convince drivers to cooperate into a more general FCD-based cooperative traffic system.

It is not the objective of this paper to analyze the subscriber base dynamic, yet some hypothesis can be made. The system presents itself very attractive for the first drivers that adopt the system since they would be given green priority on other drivers. If the number of subscribers of the system would rise then at some point the system would not be so attractive, for users, since nonusers would take advantage of the good regulation of traffic lights without the hassle of implementing the technology on their own vehicle.

New research efforts could be devoted to the study of such users dynamic to establish the final customer base levels of equilibrium in the cases that such system would be introduced as a free or as a pay for use service.

The system presented in this paper would allow the following positive results for city administrations:

(i) Allowing a better regulation of traffic lights taking advantage of FCD data [53-56];

(ii) Gathering data from FCD that would be useful for infomobility or other applications. Providing, for drivers, the incentive in terms of reduced travel 


\begin{tabular}{|c|c|c|c|c|c|c|c|c|}
\hline \multirow{2}{*}{$\frac{\text { Maneuvers }}{1,2,3}$} & \multicolumn{8}{|c|}{ Traffic light phases: (100\% demand with 90/10 repartition) - Cycle 66 [s] } \\
\hline & 12 & 4 & & & 50 & & & \\
\hline $4,5,6$ & & & 12 & 4 & & & & \\
\hline $7,8,9$ & & & & & 7 & 4 & & \\
\hline $10,11,12$ & & & 5 & & & & 7 & 4 \\
\hline
\end{tabular}

Figure 7: Example of fixed static cycle for case A.

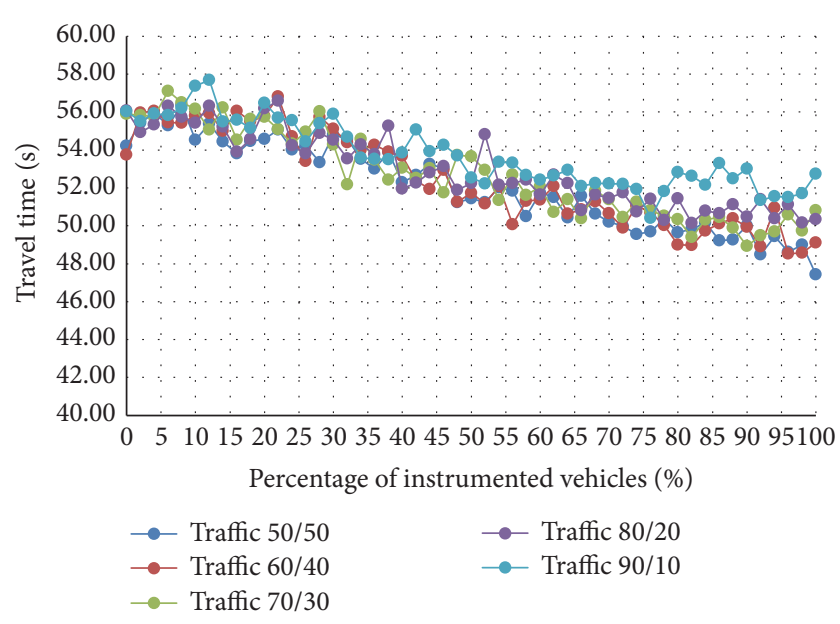

FIGURE 8: Average travel time of all vehicles (both instrumented and not instrumented) as a function of the percentage of instrumented vehicles in case A (high traffic flows).

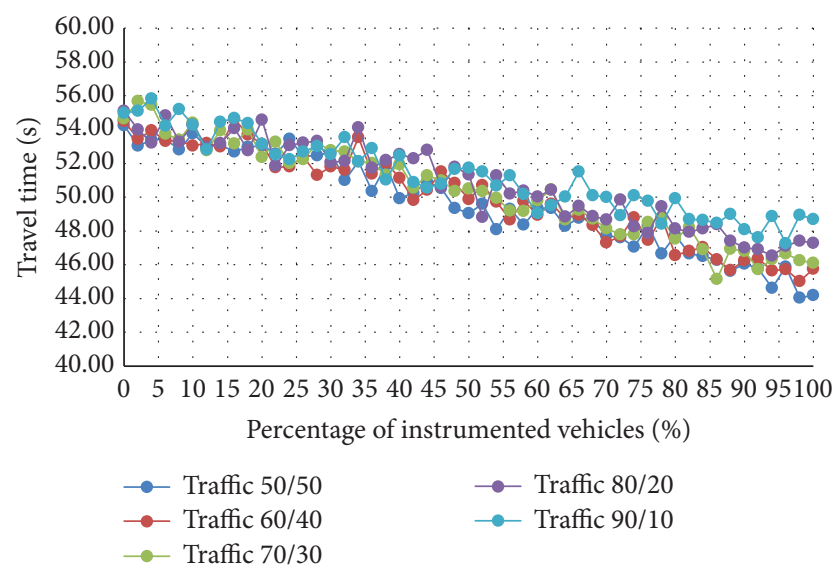

FIGURE 9: Average travel time of all vehicles (both instrumented and not instrumented) as a function of the percentage of instrumented vehicles in case B (medium traffic flows and well regulated intersection).

time to accept the proposed system as beneficial and adopt it promptly and voluntarily becoming part of a successful FCD cooperative system;

(iii) Reducing traffic congestion and pollution emissions;

(iv) Giving the administrations the justification to introduce some sort of driving toll in the case the application is introduced as a pay service.

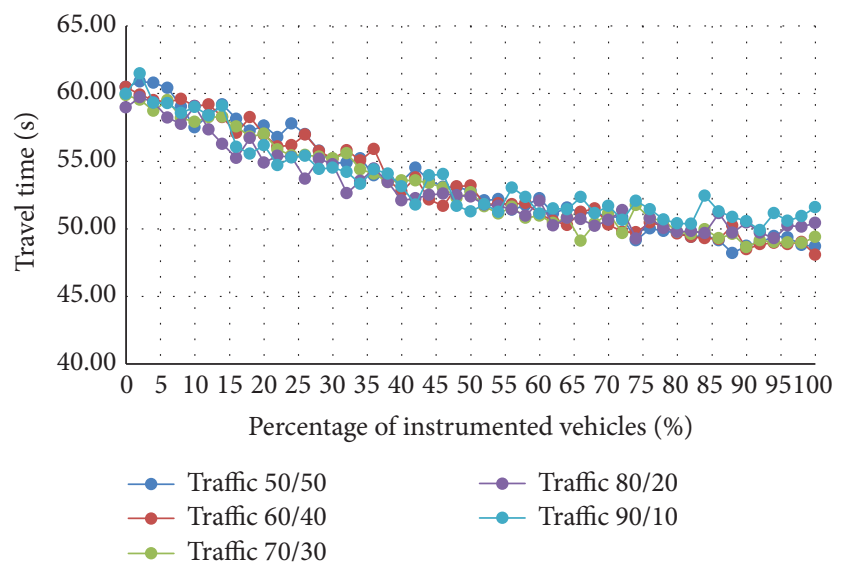

Figure 10: Average travel time of all vehicles (both instrumented and not instrumented) as a function of the percentage of instrumented vehicles in case $\mathrm{C}$ (medium traffic flows and badly regulated intersection).

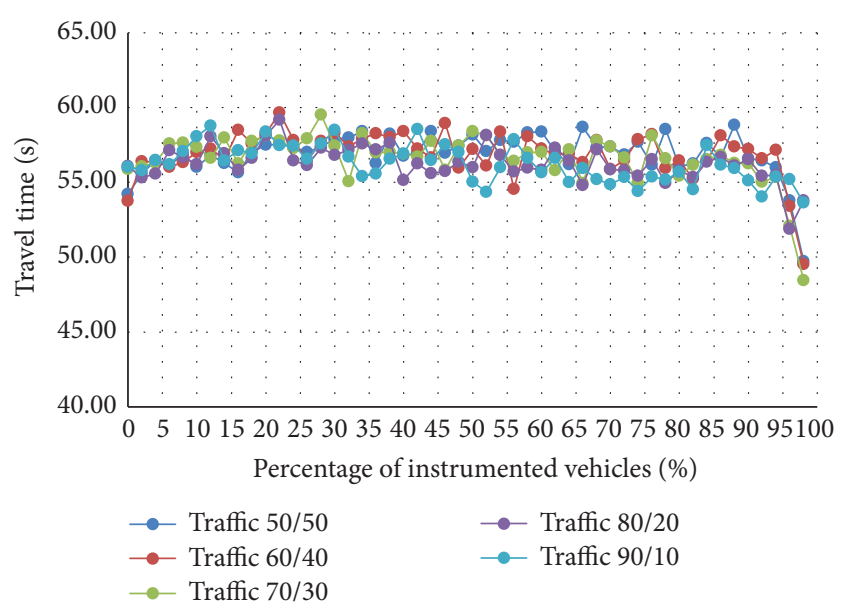

FIGURE 11: Average travel time of not instrumented vehicles as a function of the percentage of instrumented vehicles in case A (high traffic flows).

Drivers would undoubtedly take advantage of the reduced travel times at intersections that are usually poorly regulated.

This paper presents the proposed system just in general terms. It must be noted that the intersection analyzed is a simple intersection with one single lane for each approach. More complicated algorithms must be applied for more complicated intersections. 


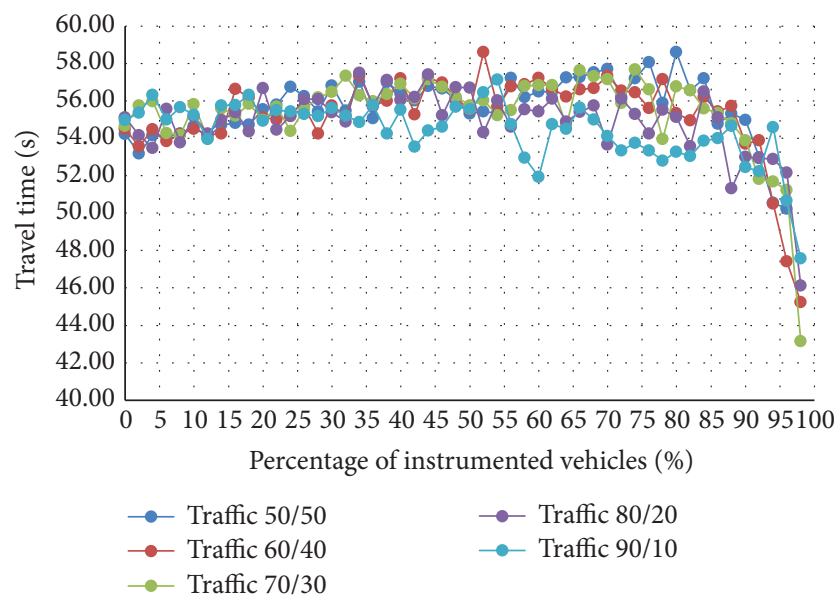

FIgURE 12: Average travel time of not instrumented vehicles as a function of the percentage of instrumented vehicles in case B (medium traffic flows and well regulated intersection).

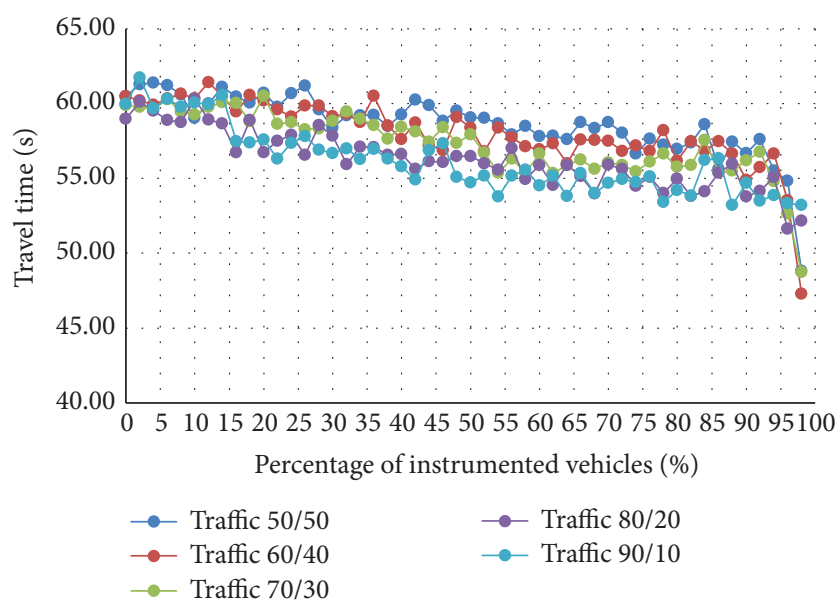

FIgURE 13: Average travel time of not instrumented vehicles as a function of the percentage of instrumented vehicles in case $\mathrm{C}$ (medium traffic flows and badly regulated intersection).

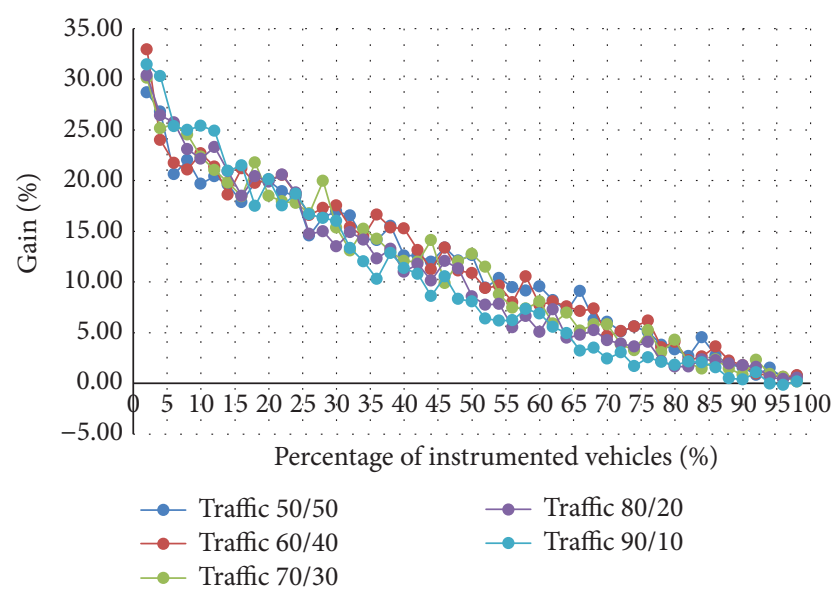

FIgURE 14: Percentage gain of travel time for instrumented vehicles relative to not instrumented vehicles in case $\mathrm{A}$.

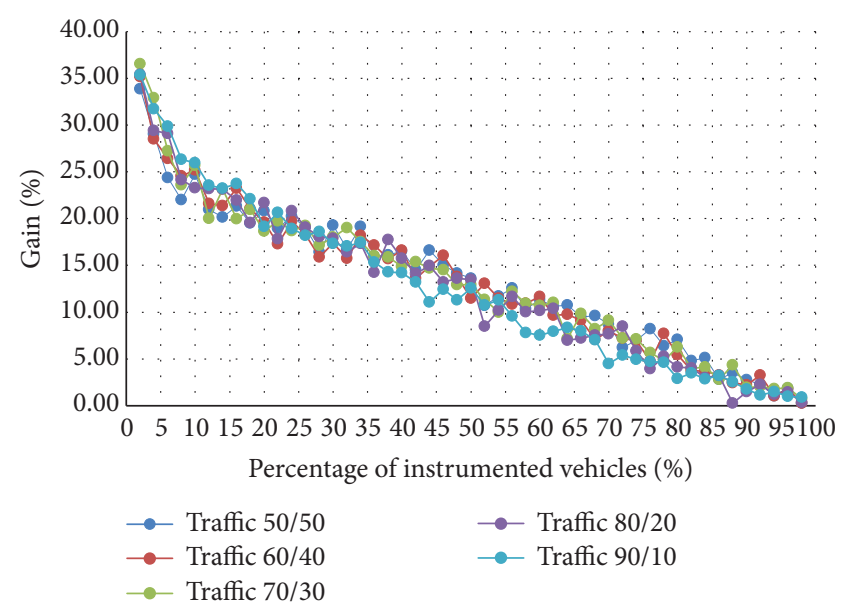

FIGURE 15: Percentage gain of travel time for instrumented vehicles relative to not instrumented vehicles in case B.

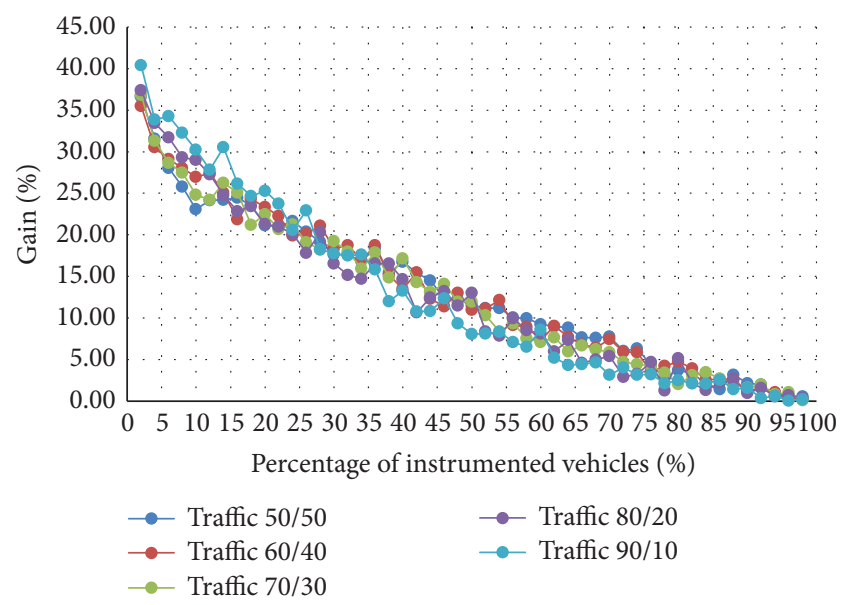

FIGURE 16: Percentage gain of travel time for instrumented vehicles relative to not instrumented vehicles in case $\mathrm{C}$.

In any case, the simulations presented in this paper show that a city administration could confidently invest in this kind of system, which would require very little infrastructure knowing that it needs a small percentage of drivers to accept this system as beneficial to guarantee that adaptive traffic signal system would perform better than prefixed cycle length.

The point of this work is to present the new concept and some of the problems connected with regulating traffic lights with FCD. This paper did not intend to be a comparison among different algorithms that instead could be analyzed in further researches.

Further research work can, in fact, be devoted to investigate more complicated intersection layouts and the control algorithms that can be applied in all possible intersection layouts and signal phase plans. 


\section{Conflicts of Interest}

The authors declare that there are no conflicts of interest regarding the publication of this paper.

\section{References}

[1] M. Tubaishat, Y. Shang, and H. Shi, "Adaptive traffic light control with wireless sensor networks," in Proceedings of the 4th Annual IEEE Consumer Communications and Networking Conference, (CCNC '07), pp. 187-191, January 2007.

[2] A. Stevanovic, J. Stevanovic, and C. Kergaye, "Green light optimized speed advisory systems," Transportation Research Record, no. 2390, pp. 53-59, 2013.

[3] G. A. Ubiergo and W.-L. Jin, "Mobility and environment improvement of signalized networks through Vehicle-toInfrastructure (V2I) communications," Transportation Research Part C: Emerging Technologies, vol. 68, pp. 70-82, 2016.

[4] T. Le, C. Cai, and T. Walsh, "Adaptive signal-vehicle cooperative controlling system," in Proceedings of the 14th International IEEE Conference on Intelligent Transportation Systems (ITSC '11), pp. 236-241, October 2011.

[5] V. Astarita, V. Gallelli, V. P. Giofrè et al., "Sistema semaforico adattivo attivato da veicoli strumentati con strumentazione fissa o mobile comprendente sistema di posizionamento satellitare (GNSS) e trasmissione dati via radio," Italy, Patent requested in April 2016.

[6] X. Ban, Y. Li, A. Skabardonis, and J. D. Margulici, "Performance evaluation of travel time methods for real time traffic applications," in Proceedings of the 11th World Conference on Transport Research, 2007.

[7] J. Wright and J. Dahlgren, Using vehicles equipped with toll tags as probes for providing travel times, California PATH Program, Institute of Transportation Studies, University of California at Berkeley, 2001.

[8] V. Astarita, G. Danieli, and S. d'Elia, "Sistema di rilievo del deflusso veicolare per il monitoraggio e controllo del traffico basato sulla registrazione della localizzazione dei terminali di telefonia mobile," Italian patent pending from 26/1/2000 and issued on 15/6/2004. Camera di commercio di Cosenza 2000.

[9] J. L. Ygnace, C. Drane, Y. B. Yim, and R. De Lacvivier, Travel time estimation on the San Francisco bay area network using cellular phones as probes. California Partners for Advanced Transit and Highways (PATH), 2000.

[10] R. Bolla and F. Davoli, "Road traffic estimation from location tracking data in the mobile cellular network," in Proceedings of the IEEE Wireless Communications and Networking Conference, vol. 3, pp. 1107-1112, September 2000.

[11] R. Bolla, F. Davoli, and F. Giordano, "Estimating road traffic parameters from mobile communications," in Proceedings of the 7th World Congress on ITS, Turin, Italy, 2000.

[12] V. Astarita and M. Florian, "The use of mobile phones in traffic management and control," in Proceedings of the IEEE Intelligent Transportation Systems, pp. 10-15, August 2001.

[13] G. Rose, "Mobile phones as traffic probes: practices, prospects and issues," Transport Reviews, vol. 26, no. 3, pp. 275-291, 2006.

[14] V. Astarita, R. L. Bertini, S. d'Elia, and G. Guido, "Motorway traffic parameter estimation from mobile phone counts," European Journal of Operational Research, vol. 175, no. 3, pp. 14351446, 2006.
[15] K. Sohn and K. Hwang, "Space-based passing time estimation on a freeway using cell phones as traffic probes," IEEE Transactions on Intelligent Transportation Systems, vol. 9, no. 3, pp. 559-568, 2008.

[16] F. Calabrese, M. Colonna, P. Lovisolo, D. Parata, and C. Ratti, "Real-time urban monitoring using cell phones: a case study in Rome," IEEE Transactions on Intelligent Transportation Systems, vol. 12, no. 1, pp. 141-151, 2011.

[17] V. Astarita, M. V. Caruso, G. Danieli et al., "A mobile application for road surface quality control: UNIquALroad," Procedia-Social and Behavioral Sciences, vol. 54, pp. 1135-1144, 2012.

[18] P. Mohan, V. Padmanabhan, and R. Ramjee, "Nericell: rich monitoring of road and traffic conditions using mobile smartphones," in Proceedings of the 6th ACM Conference on Embedded Network Sensor Systems (SenSys '08), pp. 323-336, Raleigh, NC, USA, November 2008.

[19] V. Astarita, G. Guido, D. Mongelli, and V. P. Giofrè, "A cooperative methodology to estimate car fuel consumption by using smartphone sensors," Transport, vol. 30, no. 3, pp. 307311, 2015.

[20] D. J. Dailey and F. W. Cathey, "Virtual speed sensors using transit vehicles as traffic probes," in Proceedings of the 5th IEEE International Conference on Intelligent Transportation Systems (ITSC '02), pp. 560-565, September 2002.

[21] S. Axer and B. Friedrich, "Level of service estimation based on low-frequency floating car data," Transportation Research Procedia, vol. 3, pp. 1051-1058, 2014.

[22] S. Axer and B. Friedrich, "Optimization of traffic safety on rural roads by traffic data based strategies," in Proceedings of the 13th World Conference on Transport Research, Rio de Janeiro, Brazil, 2013.

[23] F. Biral, M. Da Lio, and E. Bertolazzi, "Combining safety margins and user preferences into a driving criterion for optimal control-based computation of reference maneuvers for an ADAS of the next generation," in Proceedings of the IEEE Intelligent Vehicles Symposium, pp. 36-41, June 2005.

[24] R. Vaiana, T. Iuele, V. Astarita et al., "Driving behavior and traffic safety: an acceleration-based safety evaluation procedure for smartphones," Modern Applied Science, vol. 8, no. 1, pp. 8896, 2014.

[25] G. Guido, A. Vitale, V. Astarita, F. Saccomanno, V. P. Giofré, and V. Gallelli, "Estimation of safety performance measures from smartphone sensors," Procedia-Social and Behavioral Sciences, vol. 54, pp. 1095-1103, 2012.

[26] B. S. Kerner, C. Demir, R. G. Herrtwich et al., "Traffic state detection with floating car data in road networks," in Proceedings of the 8th International IEEE Conference on Intelligent Transportation Systems (ITSC '05), pp. 44-49, IEEE, Vienna, Austria, September 2005.

[27] M. A. Ferman, D. E. Blumenfeld, and X. Dai, "A simple analytical model of a probe-based traffic information system," in Proceedings of the IEEE Intelligent Transportation Systems, vol. 1, pp. 263-268, October 2003.

[28] J. C. Herrera, D. B. Work, R. Herring, X. Ban, Q. Jacobson, and A. M. Bayen, "Evaluation of traffic data obtained via GPSenabled mobile phones: the Mobile Century field experiment," Transportation Research, Part C: Emerging Technologies, vol. 18, no. 4, pp. 568-583, 2010.

[29] G. Guido, A. Vitale, F. F. Saccomanno et al., "Using smartphones as a tool to capture road traffic attributes," Applied Mechanics and Materials, vol. 432, pp. 513-519, 2013. 
[30] G. Guido, V. Gallelli, F. Saccomanno, A. Vitale, D. Rogano, and D. Festa, "Treating uncertainty in the estimation of speed from smartphone traffic probes," Transportation Research Part C: Emerging Technologies, vol. 47, no. 1, pp. 100-112, 2014.

[31] J. S. Wasson, J. R. Sturdevant, and D. M. Bullock, "Realtime travel time estimates using media access control address matching," ITE Journal (Institute of Transportation Engineers), vol. 78, no. 6, pp. 20-23, 2008.

[32] S. Young, Bluetooth Traffic Monitoring Technology: Concept of Operation \& Deployment Guidelines, Center for Advanced Transportation Technology, University of Maryland, College Park, Md, USA, 2008.

[33] J. Barceló, L. Montero, L. Marqués, and C. Carmona, “Travel time forecasting and dynamic origin-destination estimation for freeways based on bluetooth traffic monitoring," Transportation Research Record, no. 2175, pp. 19-27, 2010.

[34] J. Barceló, L. Montero, M. Bullejos, O. Serch, and C. Carmona, "A Kalman filter approach for the estimation of time dependent od matrices exploiting bluetooth traffic data collection," Proceedings of the TRB 91st Annual Meeting Compendium of Papers, pp. 1-16, 2012.

[35] C. Bachmann, M. J. Roorda, B. Abdulhai, and B. Moshiri, "Fusing a bluetooth traffic monitoring system with loop detector data for improved freeway traffic speed estimation," Journal of Intelligent Transportation Systems: Technology, Planning, and Operations, vol. 17, no. 2, pp. 152-164, 2013.

[36] J. Hu, M. D. Fontaine, B. B. Park, and J. Ma, "Field evaluations of an adaptive traffic signal-using private-sector probe data," Journal of Transportation Engineering, vol. 142, no. 1, Article ID 04015033, 2016.

[37] S. Axer, F. Pascucci, and B. Friedrich, "Estimation of traffic signal timing data and total delay for urban intersections based on low-frequency floating car data," in Proceedings of the International Scientific Conference Mobility and Transport Technologies, Solutions and Perspectives for Intelligent Transport Systems (Mobil. TUM '15), 2015.

[38] M. Barthauer and B. Friedrich, "Evaluation of a signal state prediction algorithm for car to infrastructure applications," Transportation Research Procedia, vol. 3, pp. 982-991, 2014.

[39] F. Saust, O. Bley, R. Kutzner, J. M. Wille, B. Friedrich, and M. Maurer, "Exploitability of vehicle related sensor data in cooperative systems," in Proceedings of the 13th International IEEE Conference on Intelligent Transportation Systems (ITSC '10), pp. 1724-1729, September 2010.

[40] P. Bellavista, L. Foschini, and E. Zamagni, "V2X Protocols for low-penetration-rate and cooperative traffic estimations," in Proceedings of the 80th IEEE Vehicular Technology Conference (VTC Fall '14), pp. 1-6, September 2014.

[41] D. Krajzewicz, R. Blokpoel, A. Bonfietti, J. Härri, S. Hausberger, and J. Dubois-Lacoste, "Traffic management based on vehicular communication at low equipment rates," in Proceedings of the 22nd ITS World Congress, paper number ITS-2826, Bordeaux, France, 2015.

[42] V. Gradinescu, C. Gorgorin, R. Diaconescu, V. Cristea, and L. Iftode, "Adaptive traffic lights using car-to-car communication," in Proceedings of the 2007 IEEE 65th Vehicular Technology Conference (VTC '07), pp. 21-25, April 2007.

[43] S. R. K. Narla, "The evolution of connected vehicle technology: from smart drivers to smart cars to self-driving cars," ITE Journal (Institute of Transportation Engineers), vol. 83, no. 7, article 22, 2013.
[44] P. Barsocchi, M. G. C. A. Cimino, E. Ferro, A. Lazzeri, F. Palumbo, and G. Vaglini, "Monitoring elderly behavior via indoor position-based stigmergy," Pervasive and Mobile Computing, vol. 23, pp. 26-42, 2015.

[45] F. Palumbo, P. Barsocchi, S. Chessa, and J. C. Augusto, "A stigmergic approach to indoor localization using Bluetooth Low Energy beacons," in Proceedings of the 12th IEEE International Conference on Advanced Video and Signal Based Surveillance (AVSS '15), pp. 1-6, Karlsruhe, Germany, August 2015.

[46] V. Astarita, V. P. Giofrè, and A. Vitale, "A cooperative intelligent transportation system for traffic light regulation based on mobile devices as floating car data (FCD)," American Scientific Research Journal for Engineering, Technology, and Sciences, vol. 19, no. 1, pp. 166-177, 2016.

[47] F. Zampella, A. R. Jimenez Ruiz, and F. Seco Granja, "Indoor positioning using efficient map matching, rss measurements, and an improved motion model," IEEE Transactions on Vehicular Technology, vol. 64, no. 4, pp. 1304-1317, 2015.

[48] N. Allurwar, B. Nawale, and S. Patel, "Beacon for proximity target marketing," International Journal of Engineering and Computer Science, vol. 5, no. 5, pp. 16359-16364, 2016.

[49] V. Astarita, G. Guido, A. Vitale, and V. P. Giofré, "A new microsimulation model for the evaluation of traffic safety performances," European Transport, no. 51, pp. 1-16, 2012.

[50] M. Van Aerde, B. Hellinga, M. Baker, and H. Rakha, "INTEGRATION-an overview of traffic simulation features," in Proceedings of the 75th Annual Meeting of the Transportation Research Board, Washington, DC, USA, 1996.

[51] V. Astarita, V. Giofré, G. Guido, and A. Vitale, "Investigating road safety issues through a microsimulation model," Procedia-Social and Behavioral Sciences, vol. 20, pp. 226-235, 2011.

[52] A. Steffen, P. Federico, and F. Bernhard, "Estimation of traffic signal timing data and total delay for urban intersections based on low frequency floating car data," in Proceedings of the International Scientific Conference on Mobility and Transport Technologies, Solutions and Perspectives for Intelligent Transport Systems (Mobil.TUM '15), Munich, Germany, June 2015.

[53] K. Al-Khateeb and J. A. Y. Johari, "Intelligent dynamic traffic light sequence using RFID," in Proceedings of the International Conference on Computer and Communication Engineering (ICCCE '08), pp. 1367-1372, May 2008.

[54] S. Sharma, A. Pithora, G. Gupta, M. Goel, and M. Sinha, "Traffic light priority control for emergency vehicle using RFID," International Journal of Innovations in Engineering and Technology, vol. 2, no. 2, pp. 363-366, 2013.

[55] T. Neumann, "A cost-effective method for the detection of queue lengths at traffic lights," in Traffic Data Collection and Its Standardization, pp. 151-160, Springer, New York, NY, USA, 2010.

[56] M. Kerper, C. Wewetzer, A. Sasse, and M. Mauve, "Learning traffic light phase schedules from velocity profiles in the cloud," in Proceedings of the 5th International Conference on New Technologies, Mobility and Security (NTMS '12), May 2012. 


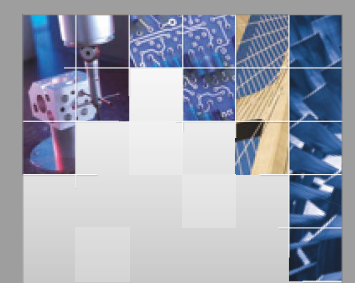

\section{Enfincering}
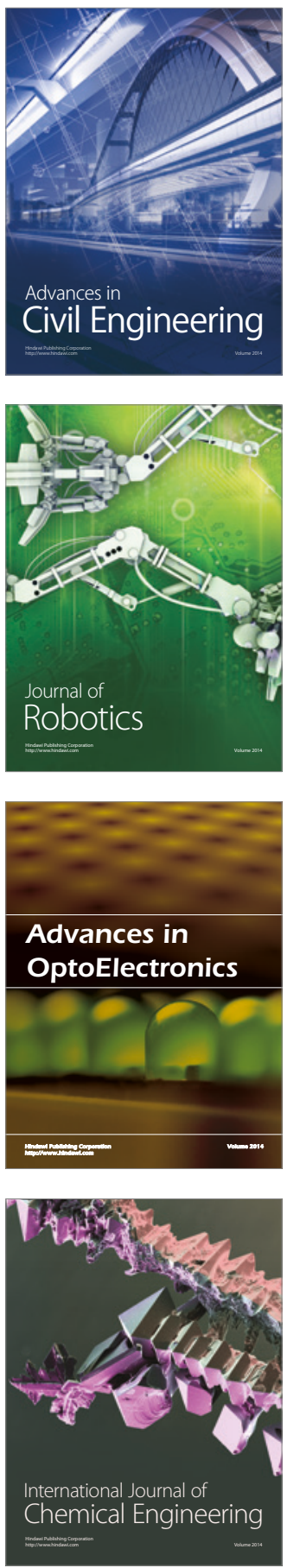

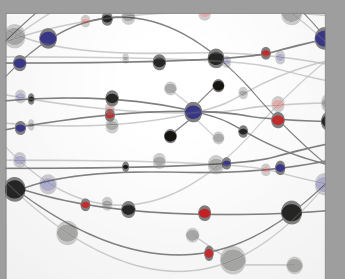

The Scientific World Journal

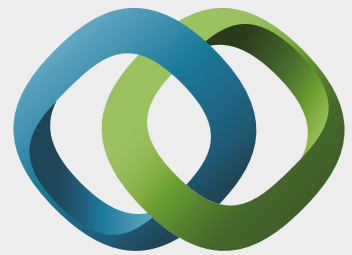

\section{Hindawi}

Submit your manuscripts at

https://www.hindawi.com
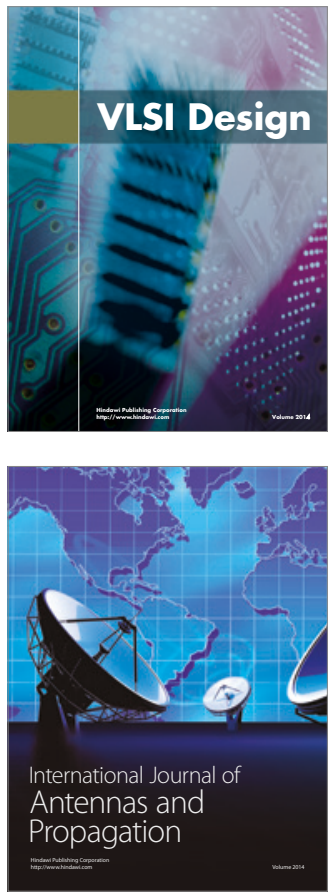

\section{Rotating}

Machinery
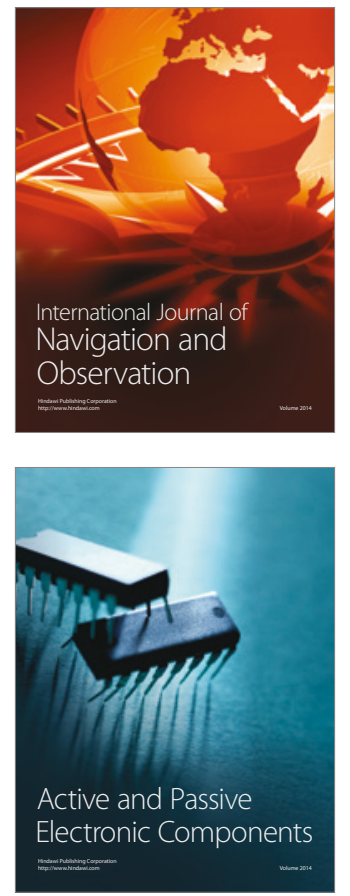
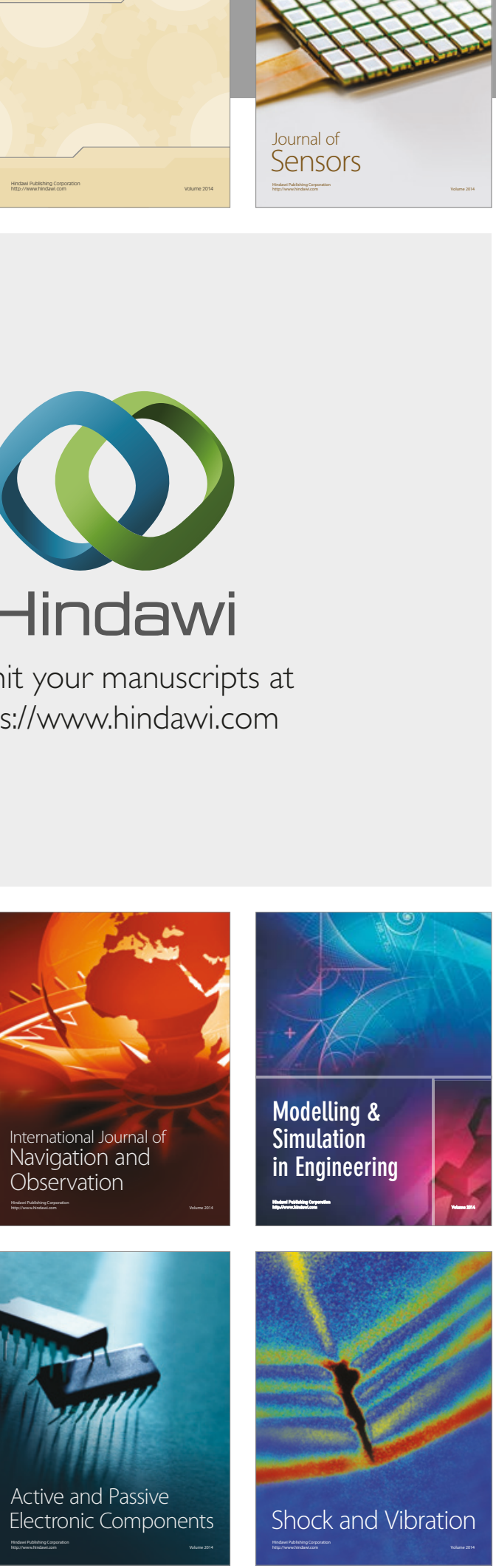
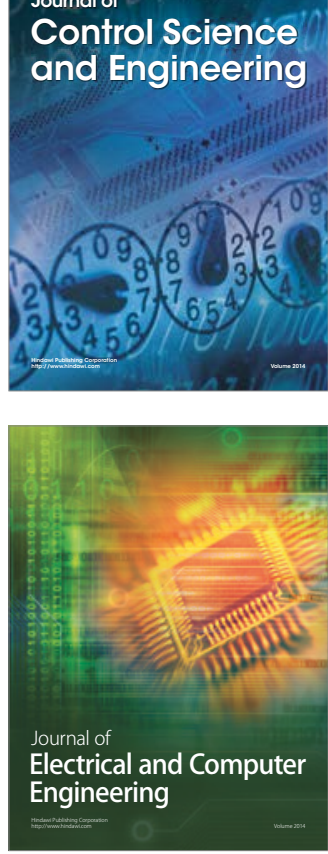

Distributed

Journal of

Control Science

and Engineering
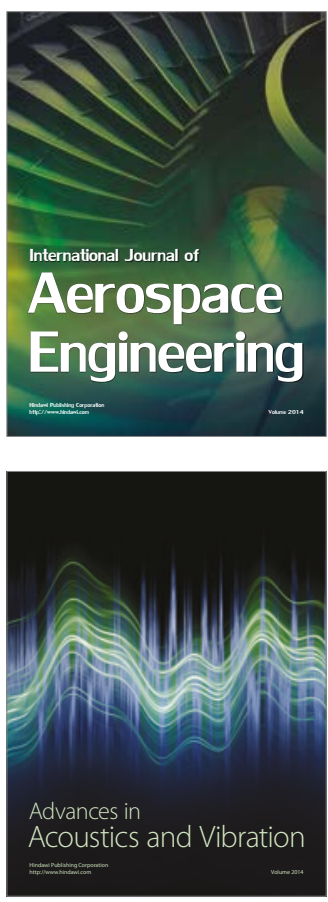

Sensor Networks 